\title{
Thelazia callipaeda ocular infection in two dogs in Belgium
}

Y. Caron, J. Premont*, B. Losson and M. Grauwels*

Department of Infectious and Parasitic Diseases, Faculty of Veterinary Medicine, University of Liège, B-4000 Liège, Belgium

*Department of Clinical Sciences, Ophthalmology, Faculty of Veterinary Medicine, University of Liège, B-4000 Liège, Belgium

Worms were retrieved from the left eyes of two dogs presented for unilateral ocular discharge in

Belgium. Morphological and molecular identification were performed and the parasites were identified as Thelazia callipaeda. The history suggested that the infection had been acquired in south-western France and southern Italy where the disease has been observed regularly for the last 6 and 12 years, respectively. In these two regions, the disease is considered endemic and spreading. To the authors' knowledge, this is the first case report of canine thelaziosis in Belgium.

Journal of Small Animal Practice (2012)

DOI: 10.1111/jsap.12003

Accepted: 2 November 2012

\section{INTRODUCTION}

Thelaziosis is caused by a parasitic nematode belonging to the genus Thelazia (Spirurida, Thelaziidae). The viviparous adult worm and larvae are found in the conjunctival fornices and nasolacrimal duct, and feed on lacrimal secretions. First-stage larvae Thelazia callipaeda are ingested by the fruit fly Phortica variegata (Diptera: Drosophilidae), which is the intermediate host found in Europe (Otranto and others 2006b). Larval development occurs in the ovarian follicles of the fly during summer. Late-stage larvae migrate to the mouthparts of the fly and are transferred to the final host when the fly feeds (Taylor and others 2007).

Thelazia callipaeda has been described in cattle, horses, cats, dogs, red foxes, wolves, European rabbits and humans (Hong and others 1995, Otranto and Traversa 2004, Otranto and others 2007, Otranto and Dutto 2008). In dogs, Thelazia californiensis in North America and T. callipaeda in Asia have been identified (Taylor and others 2007). The latter was first described in Europe, in Piedmont (Italy) 23 years ago (Rossi and Bertaglia 1989). This "oriental eyeworm" has now been detected in France (Ruytoor and others 2010), Switzerland (Malacrida and others 2008), Germany (Magnis and others 2010), Spain (Miro and others 2011), The Netherlands and Belgium (Otranto and others 2005, Janssens and Claerebout 2006).

\section{HISTORY}

A seven-year-old, spayed female golden retriever was presented to the University of Liège with a 5-month history of intermittent dis-

60 charge from the left eye (OS). Two days before presentation, the

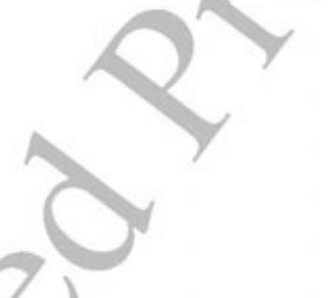

owner noticed a redness with a purulent discharge OS. This dog had been living in the Lot department in the southwest of France for several years before moving to Belgium. The second patient, an 11-year-old entire male Labrador retriever, was presented with a 3-month history of discharge OS. In the past 3 years, this dog had travelled annually to southern Italy (Basilicata), and once to the northeast of France (Alsace).

\section{Clinical examination}

The clinical examination of both dogs was unremarkable. The female was on treatment for hypothyroidism with $300 \mu \mathrm{g}$ of 1-thyroxine twice daily (Forthyron 200; Eurovet Animal Health). A Schirmer tear test (Schirmer Tear Test; Schering-Plough Animal Health Corp.) was 22 and $20 \mathrm{~mm} /$ minute in the left and right eyes of the male $\operatorname{dog}$ and 25 and $21 \mathrm{~mm} /$ minute in the female dog, respectively. In the female, purulent conjunctivitis was diagnosed OS, with severely hyperaemic palpebral and bulbar conjunctivae covered with large lymphoid follicles. In the male, mild follicular hyperplasia was present on the bulbar aspect of the nictitating membrane OS. In both patients, four threadlike motile white parasites were observed in the conjunctival fornices OS (Fig 1). Fluorescein testing (Fluorescein; Haag-Streit International) was negative in both dogs. Bilateral nuclear sclerosis was present in both dogs. The male dog had a translucent iris cyst in the left anterior chamber. Bilateral small foci of retinal dysplasia in the tapetal area were present in the female with small posterior polar subcapsular lenticular opacities OS. The rest of the ocular examination was within normal limits in both dogs.

\section{Treatment and outcome}

\begin{tabular}{|l|c|c|c|l|l|l|}
\hline \multirow{2}{*}{ LASER__WORDS } & JSAP & JSAP_12003 & \multirow{2}{*}{$\mathbb{B}$} & Dispatch: 27/11/12 & Journal: JAP & CE: \\
\cline { 2 - 3 } \cline { 5 - 7 } & Journal Name & Manuscript No. & \multirow{2}{*}{ Author Received: } & No. of pages: 44 & TS: Vijayan \\
\hline
\end{tabular}



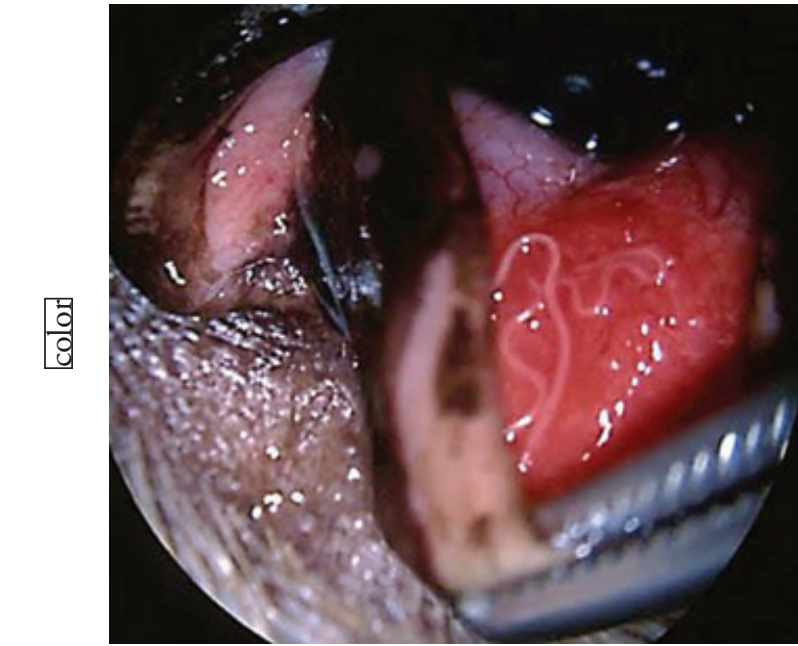

FIG 1. Thelazia callipaeda on the inflamed conjunctival bulbar surface of the nictitating membrane

$\mathrm{mg} / \mathrm{mL}$ oxybuprocaine hydrochloride $(0.5 \%$ Unicaine; Thea Pharma) was instilled before parasite removal. The specimens were collected in $70 \%$ ethanol for parasitologic identification. Both patients were treated systemically with one dose of spot-on dermal application of $10 \%$ imidacloprid and $2.5 \%$ moxidectin (Advocate Spot-On; Bayer HealthCare) and topically with a 1 $\mathrm{mg}$ dexamethasone sodium phosphate and $4 \mathrm{mg} / \mathrm{mL}$ chloramphenicol (Deicol; Meda Pharma) solution thrice daily OS for 4 weeks.

Four weeks later, the infection had resolved in both dogs. No parasites were observed. However, in the female dog, mild follicular conjunctivitis persisted; therefore, the drops were continued for 2 further weeks. One month later, both owners reported by telephone that the eyes appeared normal.

\section{Morphologic and molecular identification}

Five female worms $(15.5 \pm 2.5 \mathrm{~mm}$ long and $435 \pm 50 \mu \mathrm{m}$ wide at the widest point) and one male worm $(10.2 \mathrm{~mm}$ long and
$360 \mu \mathrm{m}$ wide at the widest point) were identified microscopically. All specimens were identified as T. callipaeda according to their 58 size, the presence of a buccal capsule, the transversally striated 59 cuticle, the position of the vulva located anterior to the oesoph- 60 agus-intestinal junction and the presence of numerous rounded 61 first-stage larvae in the distal uterus in the female worms and the 62 presence of two dissimilar spicules in the caudal bursa of the male 63 worm (Otranto and others 2003b) (Fig 2).

Molecular identification was performed with the worms collected as previously reported (Otranto and others 2005). Briefly, genomic DNA was isolated from the worms using QIAmp DNA Mini Kit (Qiagen $\mathrm{GmbH})$. The cytochrome c oxidase subunit 1 (cox1) (689 bp) was amplified using described primers and a commercial kit (Taq PCR Master Mix; Qiagen GmbH). The amplification products were purified using a commercial kit $\left(\mathrm{MSB}^{\circledR}\right.$ Spin PCRapace; Invitek) and sequenced with a genetic analyser (ABI PRISM ${ }^{\circledR} 3100$; Applied Biosystem) and compared with the BLASTn genomic database (McGinnis and Madden 2004). The coxl sequences obtained were identical to the sequence representing haplotype 1 (h1) (GenBank accession number AM042549) (Otranto and others 2005).

\section{DISCUSSION}

Both dogs affected by ocular thelaziosis were living in Belgium when diagnosed, but had travelled and stayed in various regions of southern Europe. In a previous study, T. callipaeda was identified from a dog living in the Netherlands, which had spent 3 months in the Dordogne department (Otranto and others 2005). There is another report describing a case of canine Thelaziosis in Belgium but the information included is limited, although it was noted that the dog had travelled to the Lombardia region (Italy) (Janssens and Claerebout 2006). In western Europe, canine thelaziosis is now considered endemic and widespread in south-western France (Dordogne department, close to the Lot) (Dorchies and others 2007), in southern Switzerland and all of 
1 Italy, with a prevalence as high as $60 \%$ in the Basilicata region

2 (Otranto and others 2003a).

3 The potential introduction and establishment of T. callipae-

$4 d a$ in Belgium would depend on the presence of the fly vector.

5 According to a previous study (Otranto and others 2006a) based

6 on a predictive geoclimatic model, the vector, $P$. variegata, would

7 be able to survive and multiply in Belgium. However, the pres-

8 ence of $P$. variegata has not yet been recorded in Belgium (Royal

9 Belgian Institute of Natural History, http://www.species.be).

10 The disease can be subclinical or symptomatic, with 15.4 to

$1181.4 \%$ of infected dogs showing clinical signs (Malacrida and

12 others 2008, Miro and others 2011). Affected dogs typically pres-

13 ent with follicular conjunctivitis, a mucoid to purulent discharge

14 and lymphoid tissue hyperplasia, as observed in the present cases

15 (Ruytoor and others 2010). Conjunctival petechiae and oedema,

16 epiphora (Miro and others 2011), keratitis and corneal ulcers are

17 less frequently described (Dorchies and others 2007). Clinical

18 signs may result from the mechanical damage to the ocular sur-

19 faces by the cuticle and parasite movement (Otranto 2011). The

20 foreign body sensation can lead to self-mutilation and secondary

21 infection of the eyelids, conjunctiva and cornea. Epiphora can

22 result from nasolacrimal duct obstruction by the parasites (Jans-

23 sens and Claerebout 2006). In dogs, the severity of symptoms

24 did not appear to correlate with the number of worms found

25 (Miro and others 2011). Because of the similarity in clinical

26 signs, thelaziosis should be included in the differential diagnosis

27 of infectious or allergic conjunctivitis, dacryocystitis and keratitis

28 (Otranto 2011).

29 A diagnosis is made by finding the adult worms on the ocu-

30 lar surfaces, as observed in the present cases and/or in the naso-

31 lacrimal ducts. Diagnosis can be difficult when most parasites

32 are in a larval stage, when few adult nematodes are present or

33 when parasites are located within the excretory ducts of the lac-

34 rimal glands. The latter location has not been described in dogs

35 to the author's knowledge. Identification of the worms can be

36 performed by microscopic and molecular examination. Mito-

37 chondrial genes such as the $\operatorname{cox} 1$ have proven useful for such

38 investigations because of the relatively rapid evolutionary rates of

39 these genes and the availability of gene sequences for filaroids in

40 databanks (Hu and others 2004). In this study, the use of both

41 techniques led to the identification of the same species: T. cal-

42 lipaeda ( $\operatorname{cox} 1 \mathrm{~h} 1)$.

43 Treatment of the condition is by removal of the worms. Topi-

44 cal corticosteroids and antibiotics can be used to treat the associ-

45 ated conjunctivitis and prevent bacterial contamination. A single

46 dose of $10 \%$ imidacloprid and $2.5 \%$ moxidectin by spot-on der-

47 mal application (Advocate Spot-On ${ }^{\circledR}$; Bayer) has previously been

48 shown to be effective (Bianciardi and Otranto 2005, Janssens and

49 Claerebout 2006). These treatment regimes were performed in

50 the present cases. One percent moxidectin eye drops in an aque-

51 ous solution, administered as a single dose, was also highly effi-

52 cient and well tolerated in infected dogs (Lia and others 2004).

53 In the southwest of France and the northwest of Italy, four

54 cases of human T. callipaeda infection were diagnosed (Ruytoor

55 and others 2010). Wild fauna, particularly red foxes and hares,

56 probably plays a role in maintaining and spreading the nematode amongst humans and pets in rural areas (Ruytoor and others 57 2010). However, human thelaziosis is considered a neglected 58 disease. This could be due to its high prevalence in socio-eco- 59 nomically disadvantaged communities and the lack of aware- 60 ness amongst physicians across Europe concerning the zoonotic 61 potential of this parasite (Shen and others 2006).

To the authors' knowledge, this is the first case report of 63 canine Thelaziosis in Belgium confirmed by microscopic and 64 molecular identification. With the presence of definitive hosts 65 and an increasing number of dogs travelling to and coming from 66 southern endemic regions, the establishment of T. callipaeda in 67 larger areas of Europe is possible. Further studies are required 68 to explore the vectorial capacity of Phortica spp. in northern 69 Europe, especially as the threat of global warming and climatic 70 change increases.

\section{Conflict of interest}

None of the authors of this article has a financial or personal 74 relationship with other people or organisations that could inap- 75 propriately influence or bias the content of the paper. $P$

\section{References}

Bianciardi, P. \& Otranto, D. (2005) Treatment of dog thelaziosis caused by Thelazia callipaeda (Spirurida, Thelaziidae) using a topical formulation of imidacloprid $10 \%$ and moxidectin $2.5 \%$. Veterinary Parasitology $129,89-93$

Dorchies, p. Chaudieu, G. Simeon, L. A., Cazalot, G., Cantacessi, C. \& Otranto, D. (2007) First reports of autochthonous eyeworm infection by Thelazia callipaeda ( (Spirurida, Thelaziidae) in dogs and cat from France. Veterinary Parasitology
$149,294-297$

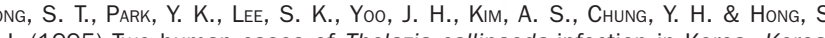
J. (1995) Two human cases of Thelazia callipaeda infection in Korea. Korean Journal of Parasitology 33, 139-144

Hu, M., Chilton, N. B. \& GAsser, R. B. (2004) The mitochondrial genomics of parasitic nematodes of socio-economic importance: recent progress, and implications for population genetics and systematics. Advances in Parasitology $\mathbf{5 6}$ 133-212

JANSSENS, G. \& ClaEREBout, E. (2006) European College of Veterinary Ophthalmologists and European Society of Veterinary Ophthalmology Congress, abstract 39 First case of dacryocystitis due to Thelazia callipaeda in a dog in Belgium. Veterinary Ophthalmology 9, 426-434

Lia, R. P., Traversa, D., Agostini, A. \& Otranto, D. (2004) Field efficacy of moxidectin 1 per cent against Thelazia callipaeda in naturally infected dogs. Veterinary Record 154, 143-145

Magnis, J., Naucke, T. J., Mathis, A., Deplazes, P. \& Schnyder, M. (2010) Local transmission of the eye worm Thelazia callipaeda in southern Germany. Parasitology Research 106, 715-717

Malacrida, F., Hegglin, D., Bacciarini, L., Otranto, D., Nageli, F., Nagel, C., Bernasconi, C., Scheu, U., Balli, A., Marenco, M., Togni, L., Deplazes, P. \& Schnyder, M. (2008) Emergence of canine ocular Thelaziosis caused by Thelazia callipaeda in southern Switzerland. Veterinary Parasitology 157, 321-327

McGINNIS, S. \& MADDEN, T. L. (2004) BLAST: at the core of a powerful and diverse se McGINNIS, S. \& MADDEN, T. L. (2004) BLAST: at the core of a powerful and
of sequence analysis tools. Nucleic Acids Research 32, W20-W25

Miro, G., Montoya, A., Hernandez, L., Dado, D., Vazquez, M. V., Benito, M., Villagrasa, M., BrIANtI, E. \& Otranto, D. (2011) Thelazia callipaeda: infection in dogs: a new parasite for Spain. Parasites \& Vectors 4, 148

OtRanto, D. (2011) Thelazia callipaeda Eyeworm: a "neglected" CVBD of human concern. Second International Conference of Southeastern and Eastern European Parasitological Society. Zagreb, Croatia, June 13-15, 2011.

Otranto, D. \& Dutto, M. (2008) Human thelaziasis, Europe. Emerging Infectious Diseases 14, 647-649

Otranto, D. \& TRAVERSA, D. (2004) Molecular characterization of the first interna transcribed spacer of ribosomal DNA of the most common species of eyeworms transcribed spacer of ribosomal DNA of the most common spe
(Thelazioidea: Thelazia). Journal of Parasitology 90, 185-188

(Thelazioidea: Thelazia). Journal of Parasitology 90, 185-188
Otranto, D., Ferroglio, E., Lia, R. P., Traversa, D. \& Rossi, L. (2003a) Current status and epidemiological observation of Thelazia callipaeda (Spirurida, Thelaziidae) in dogs, cats and foxes in Italy: a "coincidence" or a parasitic disease of the Old Continent? Veterinary Parasitology 116, 315-325

Otranto, D., Lia, R. P., Traversa, D. \& Giannetto, S. (2003b) Thelazia callipaeda (Spirurida, Thelaziidae) of carnivores and humans: morphological study by light and scanning electron microscopy. Parassitologia 45, 125-133

Otranto, D. Testinu, G. DE LuCA, F. Hu, M., Shamsi, S. \& GASSER, R. B. (2005) Analysis

OtRANTO, D., TESTINI, G., DE LUCA, F., Hu, M., ShAMSI, S. \& GASSER, R. B. (2005) Analysis
of genetic variability within Thelazia callipaeda (Nematoda: Thelazioidea) from of genetic variability within Thelazia callipaeda (Nematoda: Thelazioidea) from
Europe and Asia by sequencing and mutation scanning of the mitochondrial

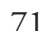
75

.




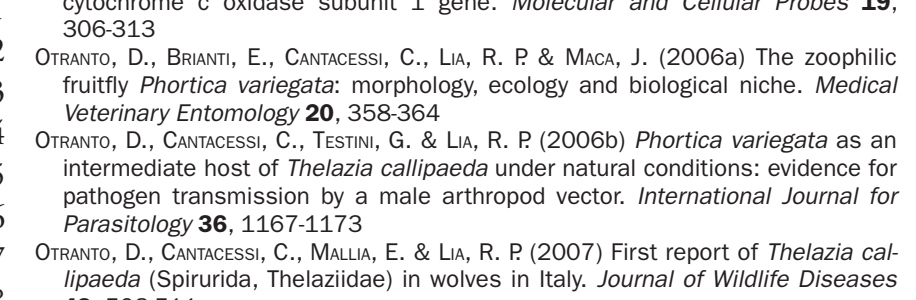

Rossi, L. \& Bertaglia, P. P. (1989) Presence of Thelazia callipaeda Railliet \& Henry, 57 1910, in Piedmont, Italy. Parassitologia 31, 167-172

Ruytoor, P., Dean, E., Pennant, O., Dorchies, P., Chermette, R., Otranto, D. \& Guillot, J. 58

(2010) Ocular thelaziosis in dogs, France. Emerging Infectious Diseases 16, 59

1943-1945

Shen, J., Gasser, R. B., Chu, D., Wang, Z., Yuan, X., Cantacessi, C. \& Otranto, D. 60 (2006) Human thelaziosis-a neglected parasitic disease of the eye. Journal of 61

TAYLoR, M. A., Coop, R. L. \& WALL, R. L. (2007) Parasites of dogs and cats. In: 62 Veterinary Parasitology. Eds M. A. Taylor, R. L. Coop and R. L. Wall. Blackwell Publishing. pp 427-428 


\section{QUERIES TO BE ANSWERED BY AUTHOR}

IMPORTANT NOTE: Please mark your corrections and answers to these queries directly onto the proof at the relevant place. Do NOT mark your corrections on this query sheet.

Queries from the Copyeditor:

AQ1 As per journal style, this statement has been added. If you do have any conflicts of interest to declare please state them when you return any proof corrections.

AQ2 Please provide the location of the publisher for the reference "Taylor and others 2007."

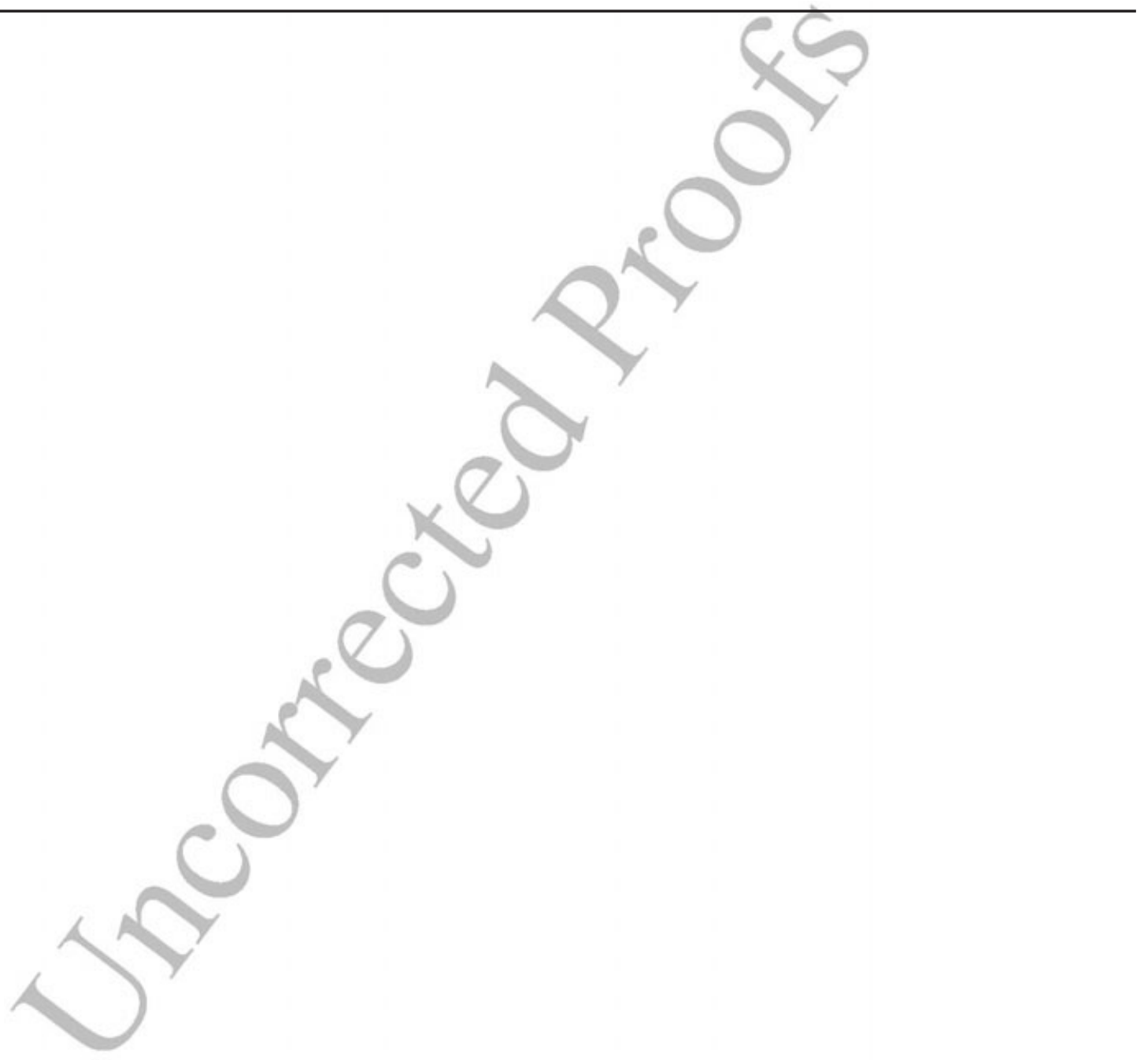


(3)WILEY-BLACKWELL

USING E-ANNOTATION TOOLS FOR ELECTRONIC PROOF CORRECTION

Required software to e-Annotate PDFs: Adobe Acrobat Professional or Adobe Reader (version 8.0 or above). (Note that this document uses screenshots from Adobe Reader $\mathrm{X}$ )

The latest version of Acrobat Reader can be downloaded for free at: http://get.adobe.com/reader/

Once you have Acrobat Reader open on your computer, click on the Comment tab at the right of the toolbar:

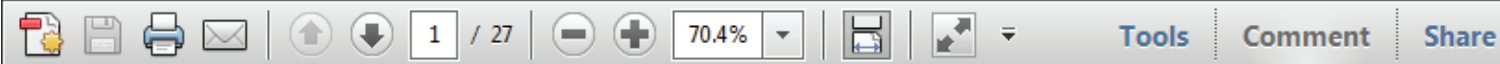

This will open up a panel down the right side of the document. The majority of tools you will use for annotating your proof will be in the Annotations section, pictured opposite. We've picked out some of these tools below:

\begin{tabular}{|c|c|c|}
\hline Annotations & & \\
\hline$\equiv$ T & 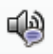 & $\Leftrightarrow$. \\
\hline $\mathbf{T}_{\boldsymbol{\Delta}} \mathbf{T}_{\widehat{Q}} \mathbf{T}$ & $\mathbf{T}$ & $\mathrm{T}$ \\
\hline
\end{tabular}

1. Replace (Ins) Tool - for replacing text.

Trikes a line through text and opens up a text box where replacement text can be entered.

\section{How to use it}

- Highlight a word or sentence.

- Click on the Replace (Ins) icon in the Annotations section.

- Type the replacement text into the blue box that appears.

Idard framework for the analysis of $\mathrm{m}$ icy. Nevertheless, it atsoled to exog

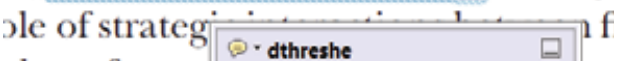
aber of comp 08/06/2011 15:58:17 ; is that the si which led nain compo: level, are ext no

which led of

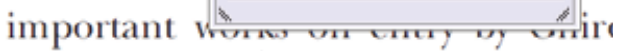
M heneforth) ' we anen the 'hlacl t

3. Add note to text Tool - for highlighting a section to be changed to bold or italic.

Tighlights text in yellow and opens up a text box where comments can be entered.

\section{How to use it}

- Highlight the relevant section of text.

- Click on the Add note to text icon in the Annotations section.

- Type instruction on what should be changed regarding the text into the yellow box that annears.

namic responses of mark ups ent with the VAR evidence

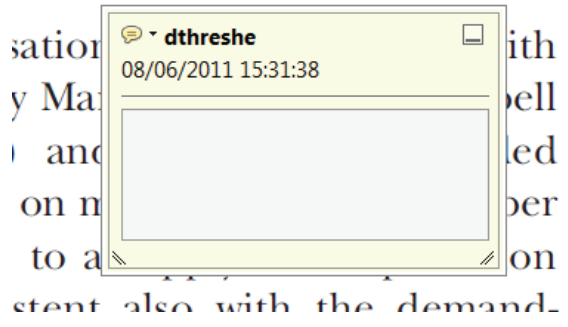

2. Strikethrough (Del) Tool - for deleting text.

Strikes a red line through text that is to be deleted.

\section{How to use it}

- Highlight a word or sentence.

- Click on the Strikethrough (Del) icon in the Annotations section.

there is no room for extra prohts al c ups are zero and the number of iet) values are not determined by Blanchard and Kiyotaki (1987), rfect competition in general equilil ts of aggregate demand and supply lassical framework assuming monol sen an evorenous number of firme

4. Add sticky note Tool - for making notes at specific points in the text.

Marks a point in the proof where a comment needs to be highlighted.

How to use it

- Click on the Add sticky note icon in the Annotations section.

- Click at the point in the proof where the comment should be inserted.

- Type the comment into the yellow box that appears.

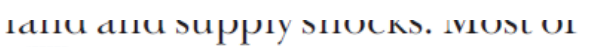

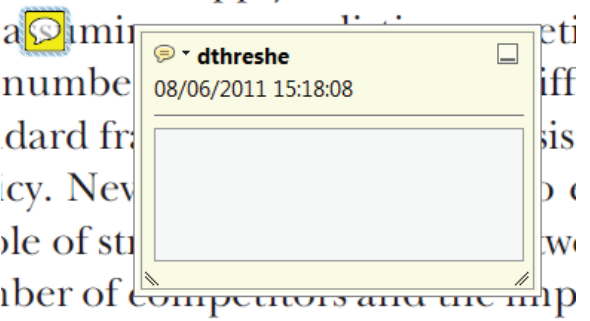

iber of eomperions anc nim inlp

is that the structure of the secto 


\section{(2)WILEY-BLACKWELL}

USING e-ANNOTATION TOOLS FOR ELECTRONIC PROOF CORRECTION

5. Attach File Tool - for inserting large amounts of text or replacement figures.

Inserts an icon linking to the attached file in the appropriate pace in the text.

How to use it

- Click on the Attach File icon in the Annotations section.

- Click on the proof to where you'd like the attached file to be linked.

- Select the file to be attached from your computer or network.

- Select the colour and type of icon that will appear in the proof. Click OK.

E N D

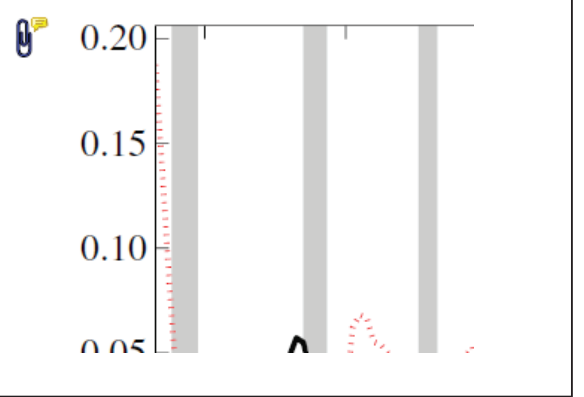

6. Add stamp Tool - for approving a proof if no corrections are required.

- Inserts a selected stamp onto an appropriate place in the proof.

How to use it

- Click on the Add stamp icon in the Annotations section.

- Select the stamp you want to use. (The Approved stamp is usually available directly in the menu that appears).

- Click on the proof where you'd like the stamp to appear. (Where a proof is to be approved as it is, this would normally be on the first page).

If the Dusiness cycie, starting with the on perfect competition, constant ret otaki (1987), has introduced produc general equilibrium models with nomin

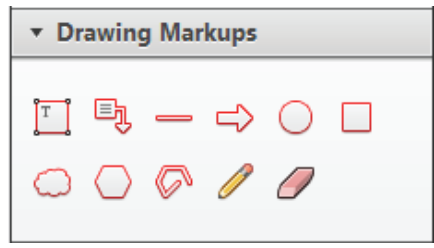

How to use it

- Click on one of the shapes in the Drawing Markups section.

- Click on the proof at the relevant point and draw the selected shape with the cursor.

- To add a comment to the drawn shape, move the cursor over the shape until an arrowhead appears.

- Double click on the shape and type any text in the red box that appears.
7. Drawing Markups Tools - for drawing shapes, lines and freeform annotations on proofs and commenting on these marks.

Allows shapes, lines and freeform annotations to be drawn on proofs and for comment to be made on these marks.

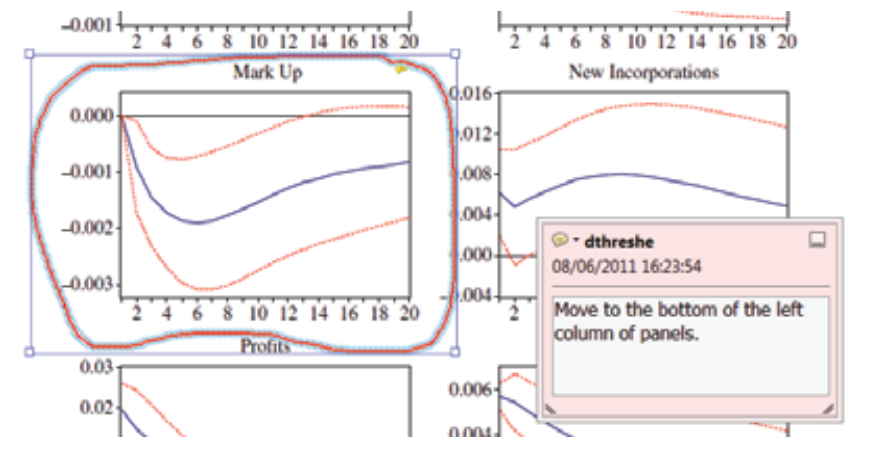

For further information on how to annotate proofs, click on the Help menu to reveal a list of further options:

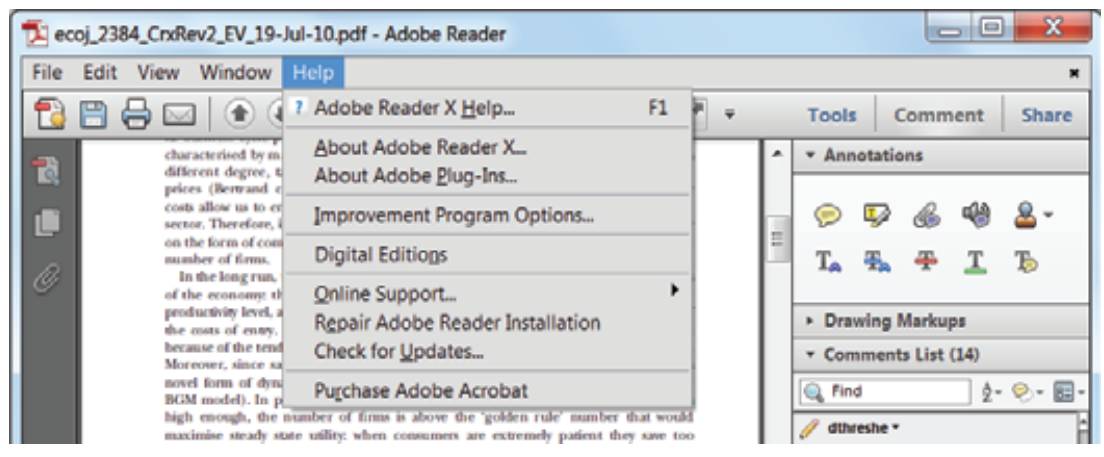

